\title{
Las agencias colombianas de publicidad como agentes del marketing nacional e internacional ${ }^{*}$
}

\author{
Adoración Merino Arribas** \\ Rafael Repiso Caballero**
}

Recibido: 2015-06-10 Enviado a pares: 2015-06-30

Aprobado por pares: 2015-07-30 Aceptado: 2015-08-04

\section{Resumen}

Este artículo es un análisis estadístico retrospectivo de la nacionalidad de dos elementos: las agencias de publicidad con sede en Colombia con mayor número de anuncios premiados en festivales nacionales e internacionales, y las marcas que presentan estos anuncios, según los datos referentes a Colombia generados por el ranking UNIR de Publicidad Iberoamericano (2012-2013). Los resultados demuestran que estas agencias son agentes del marketing nacional e internacional, destacando que son los anuncios con temática y recursos nacionales los más premiados.

Palabras clave: empresas transnacionales; globalización; agencias de publicidad; festival publicitario; Colombia.

* $\quad$ Este trabajo se enmarca en el proyecto del Ranking UNIR de Publicidad. Es una investigación financiada por el plan propio de Investigación de la Universidad Internacional de La Rioja (UNIR), bienio 2014-2016. GdI-13. Grupos COYSODI y PROCOMM.

** Doctora en Ciencias de la Información por la Universidad de La Laguna. Profesora Adjunta de la Universidad Internacional de La Rioja (UNIR) dory.merino@unir.net

*** Doctor en Documentación Científica por la Universidad de Granada. Profesor Contratado Doctor en la Universidad Internacional de La Rioja (UNIR) rafael.repiso@unir.net 


\title{
Colombian advertising agencies as domestic and international marketing agents
}

\begin{abstract}
This article presents a retrospective statistical analysis of the nationality of two elements: the advertising agencies based in Colombia with the largest number of awarded commercials within national and international festivals and the brands that these commercials represent according to the data collected by UNIR Ranking of Latin-American Advertising (2012-2013). The results show that, on the one hand, these companies are domestic and international marketing agents, and, on the other hand, the largest number of awarded commercials are those related to national resources and topics.
\end{abstract}

Key words: transnational corporations; globalization; advertising agencies; festival of advertising festival; Colombia. 


\section{Introducción}

El crecimiento sostenido de la economía de Colombia ha ocasionado que empresas multinacionales consideren a este país una zona atractiva para la inversión, lo que hace que las agencias de publicidad colombianas sean un objeto de estudio ideal para el análisis de la influencia de las multinacionales. En concreto, Colombia cerró el 2013 con un PIB (Producto Interno Bruto) del 4,3\%, un índice superior al resto de los países de América Latina y Caribe, cuyo promedio se quedó en el 3,2 \%. Siguiendo esta tónica destacamos las estimaciones publicadas por The Economist. La última tasa facilitada por el Departamento Administrativo Nacional de Estadística de Colombia (DANE), con fecha de mediados de diciembre de 2014, fija el PIB del año en un 4,7\%.

Según datos macroeconómicos, Colombia es un país cada vez más atractivo para la inversión extranjera, de acuerdo con el informe de crecimiento económico, Diamonds in the rough: Unearthing opportunity in a uncertain world 2014, citado por The Economist, que sitúa a Colombia en el sexto lugar de un ranking de 20 países con elevado crecimiento anual, quedando por debajo de China, India, Nigeria, Vietnam e Indonesia. Otro estudio, en este caso presentado por el Banco Mundial, Doing Business 2014, ubica a Colombia como el tercer destino más amigable de América Latina para hacer negocios. La pregunta que pretende resolver este trabajo es: ¿Qué papel tiene la publicidad de gran impacto y las agencias que la realizan en este proceso? O, dicho de otra forma, ¿qué participación tienen las agencias de publicidad nacionales y trasnacionales en la venta de bienes y servicios nacionales y extranjeros en Colombia?

Se entiende aquí por empresa transnacional aquella que establece su negocio en distintos países con capacidad de influir directamente en la economía mundial. Una de sus características es que contrata trabajadores naturales de los países en los que se establece, pero su mercado objetivo es global. Su sede, directivos y toma de decisiones están en el país de origen, sin que influyan demasiado las que están instaladas en el resto del mundo. Estas empresas tienen una organización jerárquica, en pirámide, con la empresa matriz en el vértice superior, con las filiales en el centro y en la base los lugares de venta. El capital, la tecnología, las decisiones y la información circulan de arriba hacia abajo (Jaramillo, Lee y Rho, 2013). En cambio, las empresas internacionales, aunque tienen sucursales en varios países su estrategia no es global -tampoco su mercado objetivo-y su cultura empresarial consiste en la adaptación de la matriz a cada circunstancia nacional en la que están presentes.

La estrategia internacional de las transnacionales se resume en transferir y adaptar el conocimiento o experiencia de la compañía matriz a los mercados extranjeros. Se busca la eficiencia global de la organización. En este proceso se construyen tres capacidades estratégicas: eficiencia y competitividad a escala global, reacción y flexibilidad en el ámbito nacional y la capacidad de cruzar distintos mercados para utilizar el aprendizaje de manera global (Bartlett y Ghoshal, 2003).

Las empresas transnacionales, en la medida en que impulsan la innovación y la internacionalización desde sus filiales, favorecen igualmente la excelencia en las activi- 
dades económicas (Álvarez y Cantwell, 2011). Una de las manifestaciones de esto son las campañas de publicidad, que influyen directamente en el consumo.

Las empresas transnacionales tienen un protagonismo clave en los procesos de globalización:

1. Son portadoras de cultura. No solo venden productos. Difunden modos de vida, estilos de consumo, aspiraciones sociales, etc. (Somavia, 1978). Merced a la publicidad de las empresas transnacionales se uniformiza el consumo, incluso en países dispares y muy diferentes en sus tradiciones.

2. Constituyen redes de intercambio sólidas que propician las relaciones políticas y económicas. Las empresas transnacionales son los principales agentes de la globalización, ya que, como portadoras de la vocación universal del capital, integran producción tecnológica, mercados, flujos comerciales y financieros, y decisiones políticas (Veloz, 2007).

3. Transforman las sociedades para convertirlas en mejores clientes. La política de imposición de la globalización se apoya en las exigencias que los Estados neocolonialistas imponen a los gobiernos, organizaciones de la sociedad civil, consumidores y élites locales con los objetivos de incrementar las ganancias del capital internacional y de generar un mayor volumen de liquidez para los mercados financieros (Añez, 2006).

No se entra aquí en el "complejo concepto de la globalización", precisamente por la multiplicidad de factores que entran en juego (Hopkins, 2002 y Martínez, 2005). Se trata simplemente de analizar una de las características de la globalización: la homogeneización de productos y la eliminación de barreras para conseguirlos y situarlos en cualquier parte del mundo. Ya en 2005, Martínez expone que la afirmación de que la Coca-Cola puede encontrarse en cualquier lugar es solo un ejemplo, pero muy significativo, de colonización -o imperialismo- cultural de Estados Unidos sobre el resto del mundo, porque está ligado a un modo propio de entender la vida y la felicidad, como poco.

Caro (2010) analiza el papel de la publicidad como plataforma imaginaria o ideología fría que actúa sobre el consumidor y concluye que constituye el elemento imaginario de la globalización. Años antes, un autor de referencia en las ciencias de la información aseguraba que la historia de la formación de las redes publicitarias internacionales es la historia de las primeras vías de acceso a la llamada modernidad mediática (Mattelart, 1989).

Las agencias transnacionales juegan un papel destacado en la globalización tanto en los aspectos económicos como en los socioculturales (Leslie, 1995). Su presencia en Colombia no es un fenómeno reciente (Leslie, 1995). Ya en 1950 se estableció Mccann Erickson (en la misma década se fortalece la radiodifusión en el país). Siguieron Leo Burnett, Ogilvy y Mather, que permanecen y que figuran en el ranking de este trabajo.

Gascue (2014), que estudia un caso similar en Uruguay desde los años ochenta, afirma que el sector de las agencias asociadas a redes transnacionales en el sistema de agencias publicitarias presenta un continuo crecimiento, en perjuicio de las nacionales. 
Un punto de vista omnicomprensivo que es frecuente en los estudios sobre publicidad parte de que "la publicidad no es un instrumento neutro y constituye el instrumento por antonomasia del capitalismo desde el que organizar la cohesión social" (Caro, 2010). De ahí se pasa a otra afirmación que exigiría un mayor nivel de análisis, ya que implica demasiadas variables al mismo tiempo:

Esta gran publicidad que fueron expandiendo urbi et orbe las agencias de publicidad transnacionales y, al frente de ellas, las estadounidenses, hacía algo más que promover la venta de productos y servicios de sus clientes, generalmente también transnacionales. Antes que ello, su primera tarea consistía en fabricar consumidores: esto es, reconducir las expectativas vitales de una mayoría de la población del área considerada, la cual accedía maravillada, gracias a la televisión, a esos fabulosos anuncios que ponían al alcance de sus ojos un modo de vida que les llegaba aureolado por el glamour de las estrellas cinematográficas que lo protagonizaban en los filmes que la factoría hollywoodiense expandía paralelamente por el mundo, de modo que sus deseos y sus ansias de modernidad se concentraran en aquellos productos miríficos -automóviles, electrodomésticos, cámaras fotográficas, cosméticos, vestuario...- que la gran producción masiva de la época ponía a la altura de los deseos de todo el mundo (p.114).

Trabajos más concretos y posteriores han matizado esta afirmación. Por ejemplo, la transnacionalidad de las agencias de publicidad se ha estudiado desde el punto de vista de la globalización comunicativa en Colombia. Algunos autores subrayan que, aunque el avance tecnológico ha creado nuevos roles profesionales en las agencias, la mayor parte de los imaginarios tradicionales sobre el trabajo publicitario permanecen incólumes en el país (García et al. 2012). Quizá por eso, Del Río y Kaufmann insisten en la importancia de investigar sobre los nuevos modelos de agencia, para que sean un aspecto relevante en los estudios científicos de publicidad.

Desde la perspectiva de los profesionales, la creatividad y la persuasión son los ingredientes básicos para que cualquier campaña publicitaria de las agencias logre sus objetivos. Según Kilgour, Sasser y Koslow (2013), la experiencia en el trabajo publicitario asegura que la creatividad premia más la originalidad que la propia estrategia. Para Rodrigo Martín (2014) la creatividad en el contexto publicitario se presenta como la herramienta que permite innovar, emprender nuevos retos, atreverse con nuevas ideas, usar la imaginación para crear y reinventarse, para hacer posibles nuevas formas de ser, de pensar, de actuar y de comunicar las causas sociales. Concluye, además, que el discurso de los valores altruistas y prosociales los demanda el público, porque confían en ellos no solamente como fuente de satisfacción personal sino como artífices de la posible reconstrucción de orden social.

Ya se ha incidido con frecuencia en que la persuasión es el elemento esencial de la publicidad. Por eso su influencia no se limita a la creación de consumidores, sino que tiene una influencia poderosa en la definición de la cultura popular, en concreto, en la norteamericana (Stuhlfaut y Yun, 2013).

Los factores emocionales se incluyen en la persuasión. "La comunicación gira a la emocionalidad. Y esta tendencia parece confirmarse como un camino casi obligado" 
(López, Ferrán, 2010, p.44). Este autor analiza si en los festivales triunfa el discurso publicitario racional o el emocional. En su análisis sobre este aspecto, concluye:

Los resultados obtenidos han concluido por confirmar la hipótesis de una evolución desde planteamientos de discursos racionales a emocionales en los últimos 20 años, en uno de los festivales iberoamericanos de comunicación publicitaria más importantes, sobre todo para el mercado español. En las conclusiones sobre la evolución del tipo de discurso publicitario en los tres festivales El Sol (1988-1998-2008) cabe destacar que sorprende comprobar el nivel de abandono del discurso racional por el emocional en los últimos 20 años. A pesar de una evolución aparentemente equilibrada, esta ha sido imparable de un polo al otro" (p. 61).

El estudio que se presenta aquí parte de los datos generados para la elaboración del "Ranking UNIR de Publicidad" que engloba a Colombia entre otros países (Repiso, 2015; Repiso y Berlanga, 2015). Está realizado sobre los premios de las agencias en festivales internacionales. Es un buen reflejo de la propia construcción del sistema: el ocupar posiciones top en los rankings de publicidad es una de las prioridades de las agencias. Los premios son símbolos de liderazgo profesional, y aumentan la reputación de la agencia que aglutina más galardones. En definitiva, evaluar los premios de una agencia significa valorar su calidad, aunque sea indirectamente (Repiso, Llorente y García, 2013). Este enfoque lo avala Yun (2014), en su estudio sobre la importancia de estos, matizados con la acción posterior a los premios que se traduce en las consultas en la red mediante buscadores (por ejemplo, Google).

En este contexto, no se puede dejar al margen la situación económica de los países emergentes. Aquí cobra fuerza la explicación de Carvalho (2010), quien afirma que una condición básica para que el sistema financiero actúe de manera funcional para el desarrollo económico en cualquier país es que se disponga de un conjunto diversificado de instituciones e instrumentos financieros capaces de satisfacer las variadas demandas vinculadas a ese proceso, así como de los objetivos de lucro de las propias instituciones financieras.

Este recorrido selectivo sobre el estado del arte constituye el contexto fundamental del doble objetivo del presente trabajo. De una parte, se pretende estudiar las agencias de publicidad colombianas de mayor éxito medido en anuncios premiados (registrados en el Ranking UNIR de Publicidad). Por otra, el origen de los productos que se publicitan servirá para ver en qué medida tienen relación con la nacionalidad o transnacionalidad de las agencias que los promovieron. La hipótesis de partida que ha guiado este trabajo afirma que las agencias de publicidad transnacionales deberían funcionar principalmente como elementos de introducción de servicios y bienes cuyo origen son empresas extranjeras, mientras que las agencias locales darán servicio principalmente a empresas nacionales.

El objetivo general de este trabajo pretende, por tanto, validar esa hipótesis, comprobar si las agencias transnacionales estudiadas publicitan mayoritariamente productos no colombianos en el entorno del país, o si destacan por un mayor número de anuncios para anunciantes nacionales, y determinar su éxito de acuerdo con los premios recabados. 
Como sub-objetivos operativos tendríamos, por tanto:

a) Determinar si las agencias transnacionales destacan por el número de anuncios premiados en festivales, en contraposición con las agencias nacionales colombianas.

b) Analizar la inversión publicitaria en Colombia en los últimos años y la evolución histórica de las agencias transnacionales y nacionales de la muestra del Ranking, con el fin de poner de relieve el papel de la publicidad como agentes del marketing nacional e internacional.

\section{Metodología}

El presente estudio es un análisis documental híbrido (cuantitativo y cualitativo) retrospectivo, pues se basa en el ranking de Agencias UNIR de Publicidad del año 2014 para las agencias con sede en Colombia, y este ranking, a su vez, se basa en los anuncios premiados en los dos años inmediatos a la edición (2012 y 2013). El ranking UNIR es un meta-producto que realiza una ponderación de los anuncios y aumenta el nivel de agregación de las agencias que los crean, según los premios que reciben en festivales de publicidad nacionales e internacionales (Repiso, Berlanga y Lloves, 2015).

Se realiza aquí un análisis sobre dos factores: las agencias de publicidad con sede en Colombia que tienen mayor número de anuncios premiados en festivales nacionales e internacionales, y en segundo lugar, la nacionalidad de las marcas a las que representan estos anuncios.

Como se ha señalado ya, los datos proceden del bruto del ranking UNIR de Publicidad de los años 2012 y 2013 (Repiso, 2015; Repiso y Berlanga, 2015). Los datos para su elaboración incluyen cuáles han sido premiados en alguno de los más de 40 festivales de publicidad de diversa índole (nacionales, iberoamericanos e internacionales) que registra. Por lo tanto, la muestra de estudio es fiable por representar a los anuncios y agencias con mayor valor para la comunidad colombiana en términos de calidad publicitaria reconocida por sus iguales; en resumen, una muestra fiable e importante que refleja fidedignamente la publicidad de mayor nivel en calidad en este país. El estudio recupera 471 anuncios, de los cuales no se ha podido identificar el origen en 52 de ellos, por lo que el análisis se concreta en 419 anuncios.

Se han seleccionado 20 agencias de publicidad colombianas y sus trabajos premiados en festivales internacionales en los años 2012 y 2013. Como criterio de selección que permita un estudio comparado se han analizado las 10 agencias nacionales y las 10 transnacionales con más premios.

Se buscaba comprobar primero qué agencias - diferenciando nacionales y transnacionales-consiguen más premios en festivales internacionales. También se ha estudiado la tipología de las marcas y los clientes que anuncian (diferenciando los nacionales de los internacionales). Además, se pretende conocer si el número de premios tiene proporción con la cantidad de anuncios realizados por cada una de ellas. Por ello se ha contabilizado 
el número de galardones que ha recibido cada agencia. Igualmente se ha identificado el origen (nacional o transnacional) de la marca publicitada en cada anuncio premiado de las agencias seleccionadas.

\section{Proceso}

1. Selección de agencias. De las 75 agencias colombianas que se han presentado a festivales internacionales que registra el ranking UNIR se han elegido 10 agencias transnacionales y otras 10 nacionales, seleccionadas por la relevancia de los premios obtenidos. El criterio ha sido el de incluir aquellas que hayan obtenido en cada caso (nacional o transnacional) un mayor número de premios en los festivales consignados.

2. El listado se ha configurado de acuerdo con el número de anuncios presentados. Esta decisión es adecuada para este estudio, pero no inhabilita la validez de otros criterios de selección.

3. Cabe destacar que se han visualizado todos los anuncios, aunque en este estudio solo es pertinente describir algunos, los que destacamos y explicamos más adelante.

4. Descarga y análisis de anuncios. Se han estudiado los 471 anuncios de las 20 agencias seleccionadas.

5. Identificación de los anuncios. Se ha procedido a la identificación del origen del producto/servicio de los anuncios.

6. Por último, se ha realizado un análisis estadístico y se han elaborado gráficos de los resultados.

7. Hemos establecido unos parámetros claros, a través de palabras clave, para relacionar los siguientes conceptos: transnacionalización-agencias-creatividad-premios. De esta forma hemos visualizado mejor los resultados.

La metodología se completa con un análisis de contenido híbrido, según el modelo de Krippendorff.

\section{Resultados}

A continuación, mostramos las 10 agencias transnacionales de publicidad más premiadas, y las 10 agencias de publicidad nacionales más premiadas, en total 20 agencias, con sus respectivos números de anuncios y galardones (tabla 1). La columna "número" de anuncios se refiere únicamente a los premiados. Da una primera idea de las dimensiones de las agencias. Responde a la relación: mayor número de anuncios distintos premiados supone unos estándares de producción probablemente más altos, mayores presupuestos y más y mejores clientes. El número de premios (y sobre todo la relación anuncios/premios) se refiere más bien a la calidad de los equipos creativos de cada agencia. Las mejor clasificadas en la primera columna constituyen una garantía general mayor para los anunciantes; las mejor situadas en la segunda y tercera hablan de una probable mayor originalidad. 
Tabla 1. Número de anuncios y premios recibidos por el Top Ten de agencias de publicidad en Colombia (nacionales e internacionales)

\begin{tabular}{c|c|c|c}
\hline \multicolumn{1}{c|}{ AGENCIA } & No ANUNCIOS & No PREMIOS \\
\hline TRANSNACIONAL OGILVY \& MATHER COLOMBIA & 70 & 178 \\
TRANSNACIONAL LOWE SSP3 & 49 & 102 \\
TRANSNACIONAL SANCHO BBDO & 45 & 84 \\
TRANSNACIONAL YOUNG \& RUBICAM COLOMBIA & 42 & 89 \\
TRANSNACIONAL LEO BURNET COLOMBIA & 40 & 51 \\
TRANSNACIONAL MCCANN ERICKSON COLOMBIA & 35 & 51 \\
TRANSNACIONAL BTL & 29 & 49 \\
TRANSNACIONAL DDB COLOMBIA & 25 & 36 \\
TRANSNACIONAL PROXIMITY COLOMBIA & 21 & 19 \\
NACIONAL & FANTÁSTICA & 19 & 17 \\
NACIONAL & LIP LTDA. & 16 & 23 \\
TRANSNACIONAL PUBLICIS COLOMBIA & 15 & 25 \\
NACIONAL & GLUE & 13 & 22 \\
NACIONAL & ON BRAND EXPERIENCE & 13 & 19 \\
NACIONAL & SISTOLE S.A. & 12 & 17 \\
NACIONAL & CVML & 10 & 12 \\
NACIONAL & DMP & 8 & 12 \\
NACIONAL & NETBANGERS & 8 & 11 \\
NACIONAL & SPHERA IMPACTA & 8 & 7 \\
NACIONAL & ATENTO & 7 &
\end{tabular}

Fuente: Elaboración propia a partir del ranking Iberoamericano de Publicidad UNIR 2014.

Por lo que se refiere a la primera hipótesis, los datos muestran que nueve de las diez agencias transnacionales se sitúan en los primeros puestos del ranking de anuncios premiados. La única excepción es la agencia transnacional Publicis Colombia, con 15 anuncios, que se desplaza del grupo creado de facto por las agencias transnacionales y se sitúa por debajo de dos agencias nacionales, Fantástica y LIP LTDA., que se posicionan por encima de ella con 19 y 16 anuncios, respectivamente. Conviene destacar que, aunque en anuncios se sitúen por delante, en premios ambas quedan por debajo de la transnacional citada (Publicis Colombia).

En resumen, las nueve primeras agencias del ranking, que son transnacionales, son las que han sacado al mercado el mayor número de anuncios premiados. Esto indica como orientación que la mayor parte del mercado publicitario colombiano (en producción de anuncios de gran calidad y probablemente de presupuesto) está en manos de las agencias transnacionales. La proporción que se deduce de estos datos no puede transmitirse sin más al conjunto de la actividad de la producción de anuncios (y por tanto, del negocio del sector), pero es indudable su carácter indicativo para el conjunto. De 485 anuncios premiados producidos en Colombia, solo 117 los produjeron agencias nacionales (24,1\% del total). No solo eso. Las agencias transnacionales se sitúan en una triple banda. Por 
delante Ogilvy\&Mather Colombia con el 14,4\%. Después cinco seguidoras que se sitúan entre el 10 y el $7 \%$, y otras tres que rondan el $5 \%$ cada una. Las primeras colombianas se sitúan ligeramente por debajo de este nivel (la mejor no alcanza el $4 \%$ ) y descienden progresivamente hasta el mínimo (no se olvide que de las mejores) como Atento que consigue el 1,4\% del total de anuncios premiados.

Esta primera referencia a los aspectos cuantitativos no agota las conclusiones. Otro aspecto de gran interés es el análisis de la relación entre anuncios premiados y premios recibidos en los certámenes internacionales que se integran en el ranking UNIR. Esta relación constituye un referente de interés para valorar la creatividad y calidad de los trabajos publicitarios presentados a concurso. Marca un índice indirecto, pero muy adecuado, para valorar la calidad en la producción de piezas de anuncios y, por lo tanto, la producción misma de estas agencias, que, no se olvide, conforman la élite de las que operan en el país.

La relación entre total de premios obtenidos en festivales y anuncios distintos premiados se mueve entre el máximo de 2,5 de Ogilvy\&Mather y el 1,4 de Mccann Erikson. En ese entorno se sitúan todas las agencias transnacionales que operan en Colombia. Tres superan el 2 y pueden considerarse el top de la calidad en producción de anuncios. Si se tiene en cuenta que las dos primeras son también las que acumulaban el mayor número de anuncios premiados puede deducirse que la calidad en los estándares de producción va de la mano de las dimensiones de las agencias. En el mundo de las agencias colombianas más importantes, los anunciantes pueden suponer que es más fácil encontrar mejores productores de anuncios en una agencia grande.

Entre las agencias nacionales, los cocientes de relación premios/anuncios se sitúan en un orden muy similar al del segundo escalón de las transnacionales. El máximo de 1,9 de GLVE es comparable al de DDB Colombia y superior al 1,8 de sancho BBDO. Salvo tres que se sitúan con una cifra de 1 , el resto tiene unos coeficientes premios/anuncios muy similares a los transnacionales. No parece, por tanto, que, salvo en el nivel de las dos más grandes y exitosas, haya grandes diferencias entre las agencias de publicidad, sean nacionales o transnacionales, en lo que a calidad de producción se refiere.

Otra cosa es el total de premios obtenidos. Esta cifra absoluta establece indudablemente otro orden: volvemos a referirnos (desde luego indirectamente) al volumen de negocio y al tamaño. Y aquí, de nuevo, como en los anuncios, lo que medimos tiene más que ver con estas variables que con la calidad y creatividad. El escalón es tan pronunciado como se comentaba ya al referirse al número de anuncios. En concreto, la que ocupa el tercer puesto, GLUE, con un total de 13 anuncios, obtiene 25 premios, que son seis más que la agencia que va en primer lugar, Fantástica, que consigue 19 premios. Incluso la agencia del segundo lugar, LIP LTDA., también está por delante, con 17 premios. Lo mismo sucede con la agencia del cuarto lugar, On Brand Experience, que supera en premios a las dos primeras del grupo de las agencias nacionales.

La segunda hipótesis se refiere a si se premian más o menos los anuncios que tratan productos o servicios colombianos o internacionales, y a qué relación hay entre esto y el carácter nacional o transnacional de la agencia que los obtiene. 
Los anuncios de productos nacionales, en color naranja, son los que han obtenido un mayor número de premios en festivales (figura 1). Tres agencias presentan productos o servicios colombianos como protagonistas de la totalidad de sus anuncios premiados. Dos agencias son nacionales (CVML y Fantástica) y una transnacional, Lowe SSP3. Hay que acentuar la importancia de esta última por ser la que cuenta con un mayor número de anuncios premiados. Del resto, en siete de ellas el porcentaje de anuncios de temática nacional es muy superior al internacional. Solo Atento, agencia nacional, se ha decantado por los productos internacionales. Esta agencia es la última en número de anuncios premiados

Figura 1. Tipología de los anuncios premiados para el Top Ten de Agencias de Publicidad de Colombia (nacionales e internacionales)

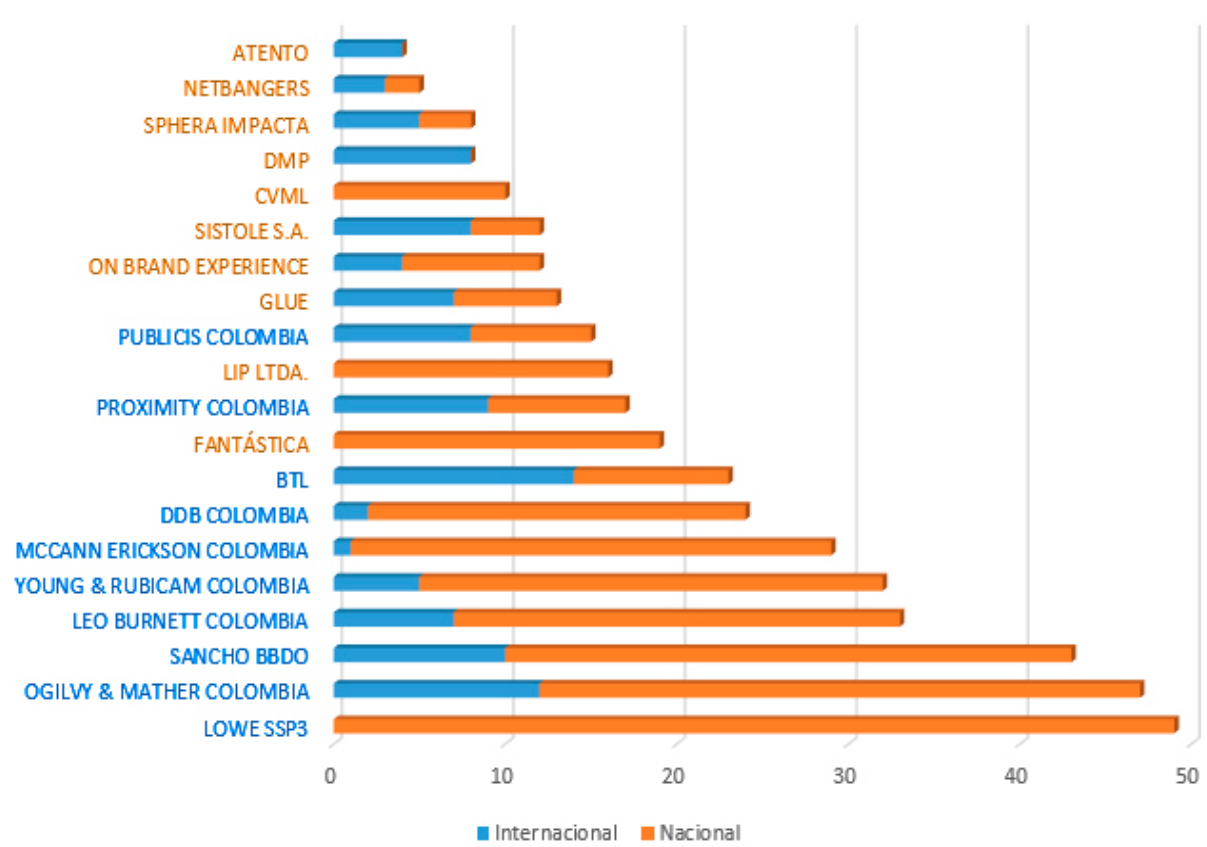

Fuente: Elaboración propia a partir del Ranking Iberoamericano de Publicidad UNIR 2014

De los 70 anuncios de la agencia mejor clasificada (OgilvyEMather Colombia), 39 -el $55,7 \%$ - se han identificado como de marcas nacionales. Otros 11 (15,7\%) son de ámbito internacional. Del resto no se ha podido identificar el producto o servicio como colombiano o internacional. Es preciso destacar que en la publicidad de marcas internacionales los anuncios seleccionados en este trabajo -teóricamente de los mejores por haber sido premiados- utilizan referentes propios de Colombia, probablemente para transmitir cercanía a los receptores. Se puede decir que este es un modo de vender su producto. Dos ejemplos de esta agencia pueden ejemplificar la tendencia. Para publicitar la Coca-Cola en Navidad, la imagen de Santa Claus reproduce el acento y las expresiones colombianas. Lo mismo sucede en el anuncio 'Autos incompletos', de la marca Allianz Seguros. Allí, dos 
personas se encuentran un mensaje en su coche aparcado en la calle con la leyenda "carro incompleto", con el consiguiente asombro por parte de los dueños del vehículo, mientras se acerca una persona con un chaleco que identifica la marca del seguro y explica que es una estrategia de la casa, provocando la risa en el dueño del vehículo.

Figura 2. Anuncios transnacionales y nacionales producidos por el Top Ten de Agencias de Publicidad

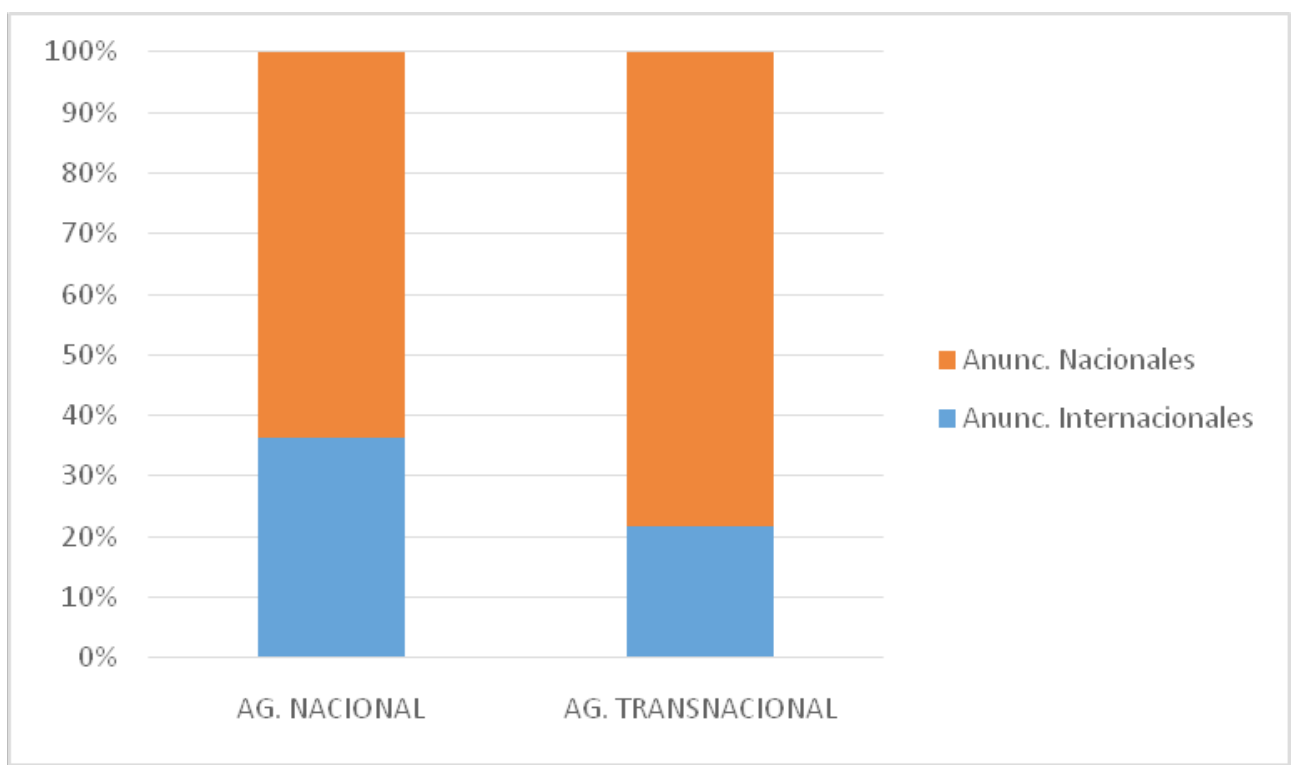

Fuente: Elaboración propia a partir del Ranking Iberoamericano de Publicidad UNIR 2014.

La agencia transnacional que destaca en publicidad de productos del país es Lowe SSP3, con 49 anuncios, todos de productos colombianos. Entre la temática publicitada destaca la ayuda social, el trueque de libros o publicidad en contra de las bebidas alcohólicas, entre otros. Esta agencia ha emitido varias campañas del Ministerio de Defensa Nacional de Colombia, con el objetivo de conseguir la desmovilización de la guerrilla. Un ejemplo de estas campañas son los anuncios Ríos de Luz o Voces en el Cielo.

Por su parte, la agencia transnacional Sancho BBDO publicita anuncios de 'orgullo colombiano', como la campaña de AviancaTaca. Además de otros con el mensaje de 'Colombia humanitaria' y los de productos de sabores tradicionales, como las papas fritas. La agencia que figura en quinto lugar, Leo Burnett, ha realizado 27 anuncios de temática nacional, de los 40 que ha presentado. Entre ellos figuran las campañas de Cruz Roja colombiana, la Liga colombiana o las Paraolimpiadas de Colombia. Otra agencia transnacional, BTL, destaca porque de los 29 anuncios que presenta, 10 son de ámbito nacional: tradiciones, show musical, chocolates y galletas de marca del país o ferias locales, entre otros.

En global, (véase la figura 2) en las agencias estudiadas domina la temática nacional en los anuncios premiados, tanto en agencias transnacionales como nacionales. Llama la 
atención que las agencias colombianas realicen más anuncios de productos extranjeros (37 \%) que las transnacionales (apenas superan el 20 \%).

\section{Discusión y conclusiones}

Destacamos como primera de las conclusiones la no correspondencia entre el resultado que comentamos en el párrafo anterior y la primera de las hipótesis. El cómputo de premios es proporcional a los anuncios que se presentan a concurso. Este dato se constata desde la primera hasta la última de las 10 agencias transnacionales. En cambio, esta tendencia se rompe en el bloque de agencias nacionales. Por tanto, efectivamente, las agencias transnacionales obtienen más premios que las nacionales en los festivales internacionales.

Al analizar las marcas extranjeras premiadas que promueven las agencias colombianas se pone de manifiesto que es Estados Unidos el país con mayor presencia publicitaria en los anuncios premiados. Por esto, los resultados muestran que las agencias colombianas de publicidad son agentes del marketing nacional e internacional. Una prueba de ello son los premios que han ganado en los festivales internacionales. Las dos primeras agencias del ranking, Ogilvy \& Mather Colombia y Lowe SSP3, superan el centenar, con 178 y 102 galardones, respectivamente.

Algunas de las marcas que aparecen en esta investigación son de productos que se consumen en todo el mundo: Nestlé, Adidas, Mercedes Benz, BMW, Audi, Ford, Smirnoff, Cadbury, Knorr, Guillete, Coca-cola, Nokia, Philips, Carrefour, Renault, Pilsen, Pepsi, Hyundai, Fanta o Sprite, entre otras. Estas marcas se pueden encontrar en cualquier parte del planeta, lo que puede considerarse uno de los efectos de la globalización, que permite la homogeneización de productos y la eliminación de las barreras para conseguirlos en cualquier parte del mundo.

Del análisis de los anuncios seleccionados para este estudio también podemos concluir que los países latinoamericanos tienen poco margen de maniobra para superar los recursos de un mercado transnacional. Algunos anuncios de las agencias nacionales promocionan productos internacionales, pero adaptándolos al país, con el fin de conseguir esa cercanía que se señalaba antes. Y, a la hora de vender lo que es puramente local se pone de manifiesto que ese anuncio no se puede exportar a otro país, porque lo que es tan localista resulta ajeno y es rechazado por el público foráneo.

Como cabía prever hay una cierta proporción entre los premios obtenidos y el número de anuncios presentado por cada agencia. Solo hay una excepción, una agencia del primer grupo, Publicis Colombia, queda después de dos nacionales, Fantástica y LIP LTDA. Ambas le superan en cuanto al total de anuncios (19 y 16, respectivamente).

Por otra parte, los anuncios con temática y recursos nacionales son los más premiados. Desde luego era lo previsible porque son los más abundantes con gran diferencia. Lo significativo es que esta preeminencia se da tanto en las agencias nacionales como en las transnacionales. Es lógico porque las agencias de publicidad buscan empatía con los potenciales compradores y para eso recurren a elementos cercanos. Se supone que 
el público rechaza lo lejano y se siente atraído por lo que tiene más cerca. Este es uno de los motivos por los que el marketing emplea elementos locales para realizar las campañas, tanto nacionales como internacionales.

Por otro lado, en esta investigación destacamos que en algunas de las campañas estudiadas se refleja cierta 'publicidad social', buscando repercusión directa en los ciudadanos. Queremos resaltar este aspecto porque pone de manifiesto la realización de campañas de comunicación por parte de administraciones, empresas privadas y todo tipo de instituciones en las que se tratan temas de interés público que pretenden concienciar, sensibilizar o informar (Alvarado y Eguizabal, 2011). La comunicación publicitaria de contenido 'social' se justifica como respuesta a las necesidades de una sociedad desarrollada, que está preocupada por la salud, el bienestar social o el medio ambiente, además de la solidaridad, la marginación, el mantenimiento de la paz, la inseguridad, el abuso en todas sus manifestaciones o la violencia, en definitiva, problemas de ámbito global. Según Alvarado (2012), se está pasando de la publicidad como institución social a la publicidad como agente social, como elemento de la acción social al servicio del cambio racional, con capacidad para contribuir a la mejora de la sociedad, de los grupos y de las personas.

Otras voces contrarias ponen de manifiesto que el modelo dominante no es solidario o, si lo es, lo es de forma disfrazada (Caro, 2010). Es más, este autor afirma que la publicidad en manos de las entidades sin ánimo de lucro tiende a mercantilizarlas. Para Caro la única salida sería una vuelta atrás: despojar a la publicidad de toda connotación comercial y devolverle su acepción comunicacional más pura la que hace referencia a publicitar en el sentido de "hacer algo público".

Agradecimientos. Agradecemos a María Ángeles Chaparro Domínguez y Julio Montero, así como a los revisores anónimos del documento por su colaboración desinteresada en este trabajo que ha supuesto una mejora sustancial del mismo.

Este trabajo se enmarca en el proyecto del Ranking UNIR de Publicidad. Es una investigación financiada por el plan propio de Investigación de la Universidad Internacional de La Rioja (UNIR), bienio 2014-2016. GdI-13. Grupos COYSODI y PROCOMM.

\section{Bibliografía:}

Alvarado, M. C. (2011). La publicidad social: una modalidad emergente de comunicación. (Tesis doctoral) Universidad Complutense, Madrid.

Alvarado, M. C. (2012). La publicidad en el marco de la comunicación para el desarrollo: hacia un nuevo modelo de publicidad para el cambio social. Cuadernos de Información y Comunicación, 17, 191-207.

Álvarez, I. y Cantwell, J. (2011). International Integratios and Mandates of Innovative Subsidiaries in Spain. International Journal of Institutions and Economies, 3 (3), 415-444.

Añez, C. (2006). Desarrollo endógeno y empresas transnacionales en la globalización. Aldea Mundo, Revista sobre Fronteras e Integración. (20). 38.Bartlett, C., Ghoshal, S. (2003). ¿Qué es un gerente global? Harvard Business Review, ISSN 0717-9952, 81, 8-87-94. 
Bartlett, C., Ghoshal, S. (2003). ¿Qué es un gerente global? Harvard Business Review, ISSN 07 17-9952, $81,8-87-94$.

Caro, A. (2010). Publicidad y globalización. Historia y Comunicación Social, (15), 117-130.

Carvalho, F. J. (2010). Reformas financeiras para apoiar o desenvolvimento.Cepal-ipea, (14) - Brasilia: Comisión Económica para América Latina y el Caribe (CEPAL)/Instituto de Investigación Económica Aplicada (IPEA).

Dabat, A., Rivera, M. y Toledo, A. (2006). Desarrollo endógeno y empresas transnacionales en la globalización. Aldea Mundo. Revista sobre Fronteras e Integración, 11(20), 37-48.

Del Río, J. y Kaufmann, J. (2014). Revisión teórica de la agencia publicitaria en la cultura de la era digital. adComunica. doi: http://dx.doi.org/10.6035/2174-0992.2014.8.5.

Diamonds in the rough: Unearthing opportunity in a uncertain world 2014. Recuperado el 26 de febrero de 2015. Disponible en: http://pages.eiu.com/Diamonds_in_the_rough_Powell.html?mkt_tok=3 RkMMJWWfF9wsRoiu6vMZKXonjHpfsX\%2B7e4oT/rn28M3109ad\%2BrmPBy73YEJWoEnZ9mMBAO ZC81kyRkK.

Doing Business (2014). Recuperado el 10 de febrero de 2015. Disponible en: http://espanol. doingbusiness.org/ /media/GIAWB/Doing\%20Business/Documents/Annual-Reports/Foreign/DB14 minibook-spanish.pdf.

García, D., Montenegro, M., Astaiza, F. y Martín, C. (2012). El campo publicitario colombiano: entre los imaginarios y las condiciones objetivas. Nómadas, 36 -255-265. Universidad Central. Bogotá. Colombia.

Gascue, A. (2014). Globalización y estructura de propiedad de las agencias publicitarias uruguayas. Cuadernos.info, (34), 103-112. doi:10.7764/cdi.34.564.

Hopkins, A. G. (2002). Globalization in World History. Londres: Pimlico.

Jaramillo, R., Lee, W., Rho, J. (2013). Análisis comparativo acerca de los diferentes roles de las filiales foráneas de empresas multinacionales chilenas y surcoreanas. Benchamaking a través de las diferencias organizacionales entre dos países. (Tesis doctoral). Universidad de Santiago de Chile.

Kilgour, M., Sasser S., and Scott K., (2013). Creativity Awards: Great Expectations? Creativity Research Journal, 25(2), 163-171. doi: 10.1080/10400419.2013.783741.

Leslie, D. (1995). The Globalization of Advertising Agencies, Concepts, and Campaigns. Economic Geography, 71 (4), 402-426.

López, R. y Ferrán, E. (2010). La evolución del discurso racional al discurso emocional en El Sol. El festival iberoamericano de la Comunicación Publicitaria (1988-1998-2008).Comunicación y Hombre: revista interdisciplinar de ciencias de la comunicación y humanidades, (6), 43-63.

Martínez, C. (2005). Globalization, audiovisual culture and international languages. Communication $\mathcal{E}$ Society 18 (1), 31-60.

Mattelart, A. (1989). La internacional publicitaria. Madrid: Fundesco.

Mattelart, A. (2003). La publicidad en la era transnacional. Ediciones Trilce. Santiago de Chile.

Universidad Internacional de La Rioja (2014). Ranking de publicidad UNIR: Recuperado el 20 de enero de 2015. Disponible en: http://rankingdepublicidad.unir.net 
Repiso, R. (2015): Premios de Publicidad Colombia 2012/2013. Figshare. http://dx.doi.org/10.6084/ m9.figshare.1309389. (14/02/2015).

Repiso, R., E Berlanga, I. (2015). Ranking UNIR, un proyecto de clasificación de la mejor publicidad iberoamercana. El profesional de la información, 24(4), 479-484. DOI: 10.3145/epi.2015.jul.15

Repiso, R, Berlanga Fernández, I., E Lloves Sobrado, B. (2015). Ranking de anuncios y agencias de publicidad en Chile.F@ro: revista teórica del Departamento de Ciencias de la Comunicación, 1(21), 5.

Repiso, R., Llorente, C., y García, F. (2013). Ranking ESCO de agencias de publicidad: descripción y resultados. El profesional de la información, 22(2), 181-187. doi:10.3145/epi.2013.mar.13

Rodrigo, I. y Rodrigo, L. (2014). La causa social en la creatividad publicitaria: Valores y confianza. Los discursos publicitarios en situaciones de crisis. Pensar la Publicidad, 7(2).

Somovia, J. (1978). La comunicación y el modelo transnacional de desarrollo.Nueva Sociedad, (38), 32-41.

Stuhlfaut, M., Yun, C. (2013). A tool for evaluating advertising concepts: Desirable characteristics as viewed by creative practitioners. Journal of Marketing Communications, 19(2),81-97, doi: 10.1080/13527266.2010.550009.

Veloz, E. (2007) Notas sobre Globalización: Las Empresas Transnacionales y el Estado en el proceso de Globalización: ¿Existe una lucha de poderes? http://www.hacienda.go.cr/centro/datos/Libro/ Notas\%20sobre\%20globalizaci\%C3\%B3n-libro.pdf. (19/04/2015).

Yun, C. (2014). Branding Potentials of Keyword Search Ads: The Effects of Ad Rankings on Brand Recognition and Evaluations. Journal of Advertising, 43(1), 85-99, DOI: 10.1080/00913367.2013.845541. 